\title{
The role of physiotherapy in the management of acute neck sprains following road-traffic accidents
}

\author{
L. A. McKINNEY, J. O. DORNAN \& M. RYAN
}

Accident and Emergency Department, Royal Victoria Hospital, Belfast, Northern Ireland

\section{SUMMARY}

In acute whiplash injuries, early physiotherapy has been shown to reduce pain and increase cervical movement, but the cost-effectiveness of this treatment has been questioned. It is unclear whether the benefits result from manipulative physiotherapy or from the patient's ability to perform the accompanying home exercise programme when instructed about its importance. In a single blind prospective randomized trial 71 patients who received out-patient physiotherapy were shown to have significant improvement in severity of neck pain $(P<0.01)$ and cervical movement $(P<0.01)$ at 1 and 2 months post-injury when compared with 33 patients who received analgesia and a cervical collar. Sixty-six patients who were offered comprehensive advice for home mobilization by a physiotherapist showed a similar improvement. There appears to be no difference in effectiveness between outpatient physiotherapy and home mobilization.

\section{INTRODUCTION}

The introduction of the compulsory use of seat-belts has been associated with an increase in the incidence of acute neck sprains (Rutherford et al., 1978). Sixty-two per cent of vehicle-occupant road-traffic accident victims develop neck pain after their accident (Deans et al., 1987), and many develop protracted symptoms which may persist beyond the settlement of litigation (Gotten, 1956). The use of cervical collars in such patients, although the mainstay of treatment $(\mathrm{McNab}, 1971)$ appears to confer little benefit (British Association of Physical Medicine, 1966). More recently it has been shown that active early mobilization is effective in improving mobility and reducing pain in the early post injury phase ( Mealey et al., 1986). Despite attempts to find prognostic factors in the initial presenting symptoms and signs (Norris \& Watt, 1983), it

Correspondence: Mr L. A. McKinney, Accident and Emergency Department, Royal Victorial Hospital, Belfast $B T 12$ 6BA, Northern Ireland. 
remains unclear whether the benefits of physiotherapy are universal and should be offered to all new patients. In Belfast's Royal Victoria Hospital, Accident and Emerg- $\widehat{0}$ ency Department, $0.5 \%$ of all new patients attend for treatment of acute neck sprains following road-traffic accidents. Treatment of all such patients with early physiotherapy would be a heavy burden on a limited service. Recently, doubt has been cast upon $\stackrel{\vec{\rho}}{\rightarrow}$ whether the beneficial effect conferred by physiotherapy is due to the relatively cost-음 effective home exercise programme instructed by physiotherapists, or to manipulative $\frac{\overline{\bar{c}}}{\bar{c}}$ physiotherapy itself (Huston, 1988). This study was designed to clarify the point by comparing the outcome for patients treated by out-patient physiotherapy, self-mobilization instructions given by a physiotherapist, and with rest and simple analgesia alone.

\section{METHODS}

With the approval of the Hospital Ethics Committee, 247 consecutive patients attend-ĩ ing the Accident and Emergency Department of the Royal Victoria Hospital, Belfast, 은 within $72 \mathrm{~h}$ of an acute flexion-extension neck sprain ('whiplash') were referred to thestudy. Having obtained informed consent, patients were entered into a single blind prospective randomized trial, and were studied over a 3-month period. The criteria for $\stackrel{\widehat{\supset}}{\square}$ exclusion were:

(1) serious cervical injury (fracture or dislocation);

(2) previously symptomatic degenerative disease; and

(3) previous whiplash injury.

Cervical spine radiography was performed on all patients. Cervical range of movementt⿳亠二口犬 and intensity of neck pain were assessed. Each patient was fitted with a soft cervical collar and was given standard analgesia (Co-dydramol tab $500 \mathrm{mg}$ ii, 6-hourly). Patients were then randomized to one of three treatment groups by sealed envelope:

Group 1. Rest and analgesia.

Group 2. Active out-patient physiotherapy.

Group 3. Mobilization advice.

\section{Group 1}

Patients allocated to this group were given general advice about mobilization after an음 initial rest period of $10-14$ days.

\section{Group 2}

Those assigned to active physiotherapy were assessed by a physiotherapist (MR) and a₹ detailed programme of out-patient physiotherapy was customized to their specifice requirements. The full gamut of physiotherapeutic aids was available as deemed疋 appropriate. Typically each patient was allocated three 40-min sessions per week for 60 weeks, and received a combination of hot and cold applications, short-wave diathermy, 
hydrotherapy, traction and active and passive repetitive movements using the principles of McKenzie \& Maitland. They were instructed about posture and exercises to perform at home. It was envisaged that this would reflect the normal practice in a general hospital physiotherapy department, and would conform to the treatment generally available to most accident and emergency departments for such patients.

\section{Group 3}

Patients assigned to the advice group were assessed by the physiotherapist, and were given verbal and reinforcing written instruction (Fig. 1) on posture correction, the use of analgesia and their collar, and instructions on the use of heat sources and muscle relaxation. Patients were encouraged to perform mobilizing exercises which were also demonstrated. This instruction session by the physiotherapist typically lasted $30 \mathrm{~min}$.

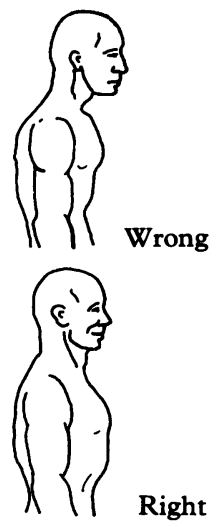

Remember POSTURE when sitting, driving, reading and standing.

Bad posture will delay your recovery.

Avoid slouching forwards with your chin sticking out.

Keep your back straight and your shoulders braced. A good exercise is to draw yourself up straight with your chin tucked in, repeatedly ten times every hour.

Getting the movements back is very important.

Here are some more exercises to help you.

FIRST straighten up - then try to touch each ear down onto your shoulder.

Straighten up again - now try looking round over each shoulder in turn.

Your exercises will be painful initially but will not harm your neck. You can repeat the exercise as often as you like. The more the better. Try to stretch your neck more each day.

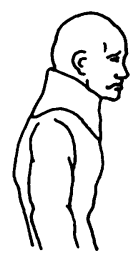

AVOID excesive reliance on a collar ... it will encourage bad posture and delay mobilization.

You will not harm your neck or delay recovery by not wearing your collar. It should only be worn for short periods at a time, or reserve it for night time use.

DISCOMFORT in your neck will gradually settle, but may be improved by local heat application - use a hot water bottle, heated towel, heat lamp or warming linament.

The pain killing tablets that you have been given can be used to supplement this.

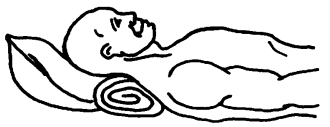

Fig. 1 Patient advice sheet.
In BED ... don't use too many pillows. Keep your neck supported either with the collar, or by making a firm roll (using a rolled up hand towel) placed inside the bottom edge of your pillow case. If possible sleep on your side or your back not on your face.

In the morning start the day with your exercises to relieve any stiffness in your neck. 
Emphasis was placed on maintaining a good range of neck movements and on correcting posture, even if this initially caused some increase in discomfort. The physiotherapist ${ }^{\circ}$ checked that the prescribed collar was correctly fitted and patients were told that use of $\frac{3}{\infty}$ the collar should be restricted to very short periods in situations where their neck was? vulnerable to sudden jolting. If a collar was worn the patient was instructed that the exercise programme should be performed immediately afterwards.

All patients reattended a neck review clinic at monthly intervals. The examining $\frac{\bar{\sigma}}{\bar{N}}$ doctor was unaware of which treatment group the patient belonged to. Information was $\frac{\pi}{\sigma}$ collected about the circumstances of the collision, perceived blame and the presence of $\triangle$ impending legal action. Range of cervical movement in the three cardinal directions was of assessed with a goniometer, and mean lateral flexion was calculated. Reduction in lateral. flexion appears to correlate well with reduction in other neck movements, and is easy to $\vec{\omega}$ measure accurately with a standard goniometer. Assessment of pain was performed using a standard $10-\mathrm{cm} 10$-point visual analogue scale with standard instructions for its $\leftrightarrows$ use (Fig. 2) (Scott \& Huskisson, 1976) . A numerical pain score was thus obtained.

Cervical movement was assessed by parametric statistical analysis using independent $\vec{\sim}$ sample $t$-tests for differences between the groups, and paired $t$-tests for differences 0 within the groups. Pain scores were assessed statistically using Mann-Whitney $U$-tests $\stackrel{?}{\rightarrow}$ for differences between groups and Wilcoxon signed rank tests for differences within $?$ groups; these being non-parametric analyses.

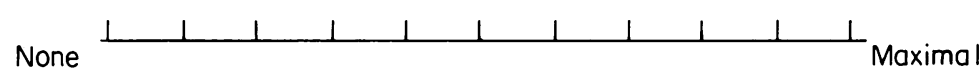

Neck Pain

Please put on $X$ on the scale at the point that you think would best describe the severity of your neck pain at present.

Fig. 2 Visual analogue scale.

\section{RESULTS}

Of the initial 247 patients who were entered in the study, 170 reattended for review at $1 \frac{1}{3}$ and 2 months post-injury, and their outcome is analysed. The 77 patients who are not included failed to attend for review. Ninety per cent of these failed to attend for their first review, the remainder on subsequent occasions. Of these, four had a full range of 9 movement when last seen and are presumed to have settled. The failed attendances are distributed evenly amongst the three groups and do not differ significantly in age, sex or $\cong$. initial severity of clinical signs from those who attended. Non-attenders were contacted $\widetilde{\sim}$ by telephone or letter on at least three occasions before being discounted.

Our information about proposed litigation is incomplete and appears inaccurate, as $\tilde{\omega}_{\mathrm{U}}$ several of those who stated that litigation was not being considered were subsequently found to have instructed a solicitor. No analysis has been attempted on the effect ofo litigation.

During the study it was felt that simple instruction about effective mobilization could $\stackrel{\text { ? }}{?}$ not reasonably be withheld, and because of this ethical objection to the rest/analgesiao 
limb it was discontinued before the conclusion of the trial; thus the numbers in this group are smaller. Seventy-one patients were allocated to the physiotherapy group and 66 to the advice group. Thirty-three patients belonged to the rest/analgesia group. The three groups were similar initially in age and sex distribution and in delay time prior to initial attendance. Fifty-two per cent of the analysed patients were male, with mean age of 30.65 years (standard deviation $10 \cdot 78$ )

Table 1 Age distribution

\begin{tabular}{lclll}
\hline & Physiotherapy & Advice & Rest & \\
\hline$n$ & 71 & 66 & 33 & $(170)$ \\
Mean age & $31 \cdot 6$ & $30 \cdot 2$ & $28 \cdot 7$ & $(30 \cdot 6)$ \\
& $(11 \cdot 3)^{*}$ & $(11 \cdot 3)^{*}$ & $(8 \cdot 9)^{*}$ & \\
\hline
\end{tabular}

*(standard deviation)

Initial mean lateral flexion was similar for all three treatment groups, but at one month post-injury both the physiotherapy and advice groups had made a significant improvement $(P<0.01)$, whereas the rest group had not. There was no difference between the improvement gained by the physiotherapy and advice groups $(P=0 \cdot 81)$. At 2 months post-injury there was no significant difference between mean lateral flexion in the physiotherapy and advice groups, and both had improved significantly when compared with the previous month $(P<0.01)$, and with the current range of movement of the rest group $(P<0.01)$.

Table 2 Neck movement (mean lateral flexion) degrees

\begin{tabular}{lccc}
\hline$n$ & $\begin{array}{c}\text { Physiotherapy } \\
71\end{array}$ & $\begin{array}{c}\text { Advice } \\
66\end{array}$ & $\begin{array}{c}\text { Rest } \\
33\end{array}$ \\
\hline Initial ROM & $45 \cdot 6(18 \cdot 5)^{*}$ & $47 \cdot 3(20 \cdot 7)^{*}$ & $44 \cdot 4(14 \cdot 7)^{*}$ \\
1 month & $53 \cdot 3(20 \cdot 3)^{*}$ & $54 \cdot 1(19 \cdot 7)^{*}$ & $41 \cdot 8(18 \cdot 9)^{*}$ \\
2 months & $64 \cdot 0(12 \cdot 9)^{*}$ & $64 \cdot 1(12 \cdot 7)^{*}$ & $55 \cdot 1(14 \cdot 8)^{*}$ \\
\hline
\end{tabular}

*(standard deviation)

Pain severity scores were initially similar for all three groups. At one month postinjury there was no difference between the physiotherapy and advice groups, but both groups were significantly better than the rest group $(P<0.01)$, which had shown no improvement over this period. There was a similar significant improvement in the

Table 3 Neck pain severity (median pain score)

\begin{tabular}{lccc}
\hline$n$ & $\begin{array}{c}\text { Physiotherapy } \\
71\end{array}$ & $\begin{array}{c}\text { Advice } \\
66\end{array}$ & $\begin{array}{c}\text { Rest } \\
33\end{array}$ \\
\hline Initial pain & $5 \cdot 32$ & $5 \cdot 3$ & $5 \cdot 6$ \\
1 month & $3 \cdot 28$ & 3.37 & 4.97 \\
2 months & 1.94 & 1.82 & 3.0 \\
\hline
\end{tabular}


actively treated groups over the following month, both of which were still comparable. A significant improvement had also occurred in the rest group, but the difference between this and the other groups was still significant $(P<0.01)$.

\section{DISCUSSION}

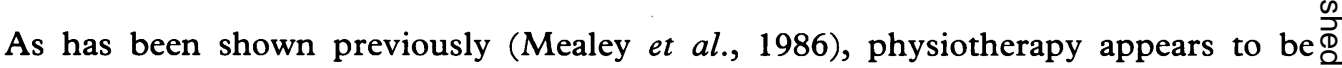
effective in improving cervical movement and reducing pain severity in the early post- ڤ injury period. However, the benefit obtained when assessed by cervical movement or $\vec{\circ}$

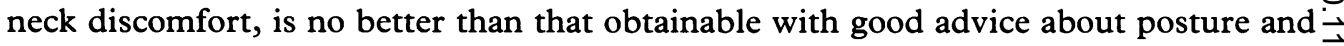
mobilization exercises, supplemented where appropriate by written instruction. The $\vec{\omega}$ improvement obtained in neck movement, compared to conventional treatment with $\frac{\stackrel{D}{\exists}}{3}$ rest and analgesia is approximately $10^{\circ}$ of lateral flexion, or a reduction of neck pain of ${ }_{\sigma}^{3}$. approximately $10-15 \%$. The recovery of those who are advised to rest will lag behind $\overrightarrow{-}$ those mobilized early by approximately $1 \mathrm{month}$. The beneficial effect, in addition to being statistically significant is clinically evident. Were all new patients to be given 을 physiotherapy, it has been calculated that in our hospital, under normal conditions each $\vec{z}$ would receive approximately $10 \mathrm{~h}$ in the physiotherapy department over a 4-6-week period. With approximately 350 new patients per year the total physiotherapy time $\stackrel{\bigcirc}{工}$ involved $(3500 \mathrm{~h})$ is equivalent to an additional requirement of two full-time physio- $\vec{\bullet}$ therapists. Providing advice and encouragement for self-help in mobilizing after ạm acute neck sprain, may allow physiotherapists to devote more time to specific problem patients with severe injury, rather than being swamped by a mass of patients wi帛 relatively minor and largely self-limiting injuries.

Efforts were made to contact the non-attenders, and they were only discounted after $\frac{\mathscr{2}}{\mathbb{Q}}$ three letters or telephone calls had produced no effect. We felt that non-attenders were $\stackrel{2}{\vec{O}}$ also more likely to have failed to follow their treatment programme, and that more $\frac{0}{3}$ strenuous efforts towards their re-inclusion in the study would thus corrupt our data. However, it is possible that the reasons for non-attendance were different between the groups - for example adequate recovery in one group and dissatisfaction and continuing $\stackrel{\mathbb{D}}{\exists}$ symptoms in another. However, this would only serve to heighten the difference between those who are actively treated and those who are not.

We conclude that good advice and tailored practical instruction on early mobilization, when given by a suitably experienced physiotherapist, is as effective as out-patient physiotherapy in reducing pain and increasing mobility and would recommend this as 을 an ideal alternative in the management of the increasing number of patients with acute $\frac{T}{2}$ neck sprains, within the constraints of limited physiotherapy resources.

\section{REFERENCES}

British Association of Physical Medicine (1966) Pain in the neck and arm: a multicentre trial of the effects of physiotherapy. British Medical fournal i, 253-58.

Deans G. T., Magalliard J. N. et al. (1987) Neck sprains-a major cause of disability following car accidents. Injury 18, 10-12. 
Gotten, N. (1956) Survey of one hundred cases of whiplash injury after settlement of litigation. Fournal of the American Medical Association 162, 865-7.

Huston G. J. (1988) Collars and corsets. British Medical fournal 296, 2756.

McNab I. (1971) The Whiplash Syndrome. Orthopaedic Clinics of North America 2, 389-403.

Mealey K., Brennan H. \& Fenelon G.C.C. (1986) Early Mobilization of Acute Whiplash Injuries. British Medical fournal 292 656-7.

Norris S. H. \& Watt I. (1983) The prognosis of Neck Injuries resulting from rear-end collisions. Fournal of Bone and foint Surgery 65b, 608-11.

Rutherford W. H., Greenfield T. et al. (1985) The Medical Effects of Seat Belt Legislation in the United Kingdom. DHSS Research Report No. 13. London, HMSO.

Scott J. \& Huskisson E. (1976) Graphic Representation of pain. Pain 2, 175-85. 